\title{
The future of PHENIX: upgrading to sPHENIX and beyond
}

\author{
E.J. Mannel ${ }^{1}$, on behalf of the PHENIX Collaboration \\ ${ }^{1}$ Brookhaven National Laboratory, PO Box 5000, Upton NY 11973
}

\begin{abstract}
PHENIX is a major upgrade to the PHENIX detector enabling high-rate, large acceptance measurements of upsilons, direct photons and fully reconstructed jets in p-p, p-A and A-A collisions at the Relativistic Heavy Ion Collider (RHIC). These detailed measurements will probe the Quark Gluon Plasma near its transition temperature, in a region of strongest coupling. The sPHENIX detector consists of hadronic and electromagnetic calorimetry, and charged particle tracking in conjunction with the recently acquired 1.5 tesla BaBar super-conducting solenoid. The sPHENIX acceptance of $2 \pi$ in azimuth and $|\eta|<1.1$ in pseudo-rapidity provides a factor of six improvement over the present PHENIX central spectrometer. Beyond being an excellent RHIC detector, sPHENIX provides an outstanding foundation for a detector focused on the physics of a possible future electron-ion collider at RHIC (eRHIC). In this talk we will discuss the physics potential of the sPHENIX detector, the design and technology choices for the sPHENIX calorimeters, and the conceptual design of a day-one detector for eRHIC.
\end{abstract}

\section{Introduction}

The PHENIX collaboration has proposed a major upgrade to the PHENIX detector, sPHENIX, to address detailed questions about the nature of the Quark-Gluon Plasma (QGP). The detector is optimized to measure jets in heavy ion collisions at the RHIC, as well as in polarized proton collisions and can form the foundation for a future eRHIC detector.

The motivation for studying jets at RHIC develops from soft sector measurements. These measurements point to a small viscosity hydro evolution that favors a strongly coupled medium which is the center of the emerging model of heavy ion collisions. In the soft sector, some models indicate a difference in shear viscosity to entropy ratio at LHC and RHIC. Although soft sector measurements continue to improve, what is missing from this is an understanding of the hard sector physics; parton energy loss, heavy quark jets and quarkonia.

As an example of how one might use measurements in the hard sector, many condensed matter systems have a minimum viscosity per unit entropy ratio near the phase transition point. While there is evidence that the QGP viscosity is very low, and near the lower limit for quantum fluids, the QGP viscosity is not well constrained. This low viscosity implies a large coupling between the constituents, and hence a greater interaction with jets. An inverse relationship between the viscosity ratio and q-hat, a measure of the inverse scattering in the medium, has been proposed by A. Majumder, B. Muller and X.N. Wang[1] as shown in Figure 1. Other aspect of hard sector physics that can be investigated in addition to jet quenching include the time dependence, $Q^{2}$, and length scale within the QGP. 


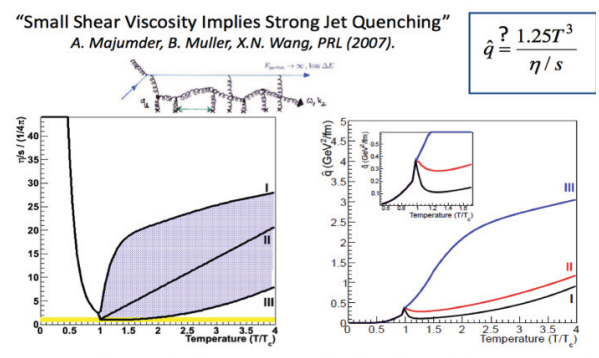

Key is independently measuring both sides of this equation!

Figure 1: The relationship of the viscosity ratio and q-hat[1].

Table 1: Projected event rates for different running conditions. The projections are based on 200 billion events sampled and 50 billion events collected in 20 weeks of $\mathrm{Au}+\mathrm{Au}$ running

\begin{tabular}{|c|c|c|c|}
\hline$-1<\eta<1$ & $\begin{array}{l}\mathrm{Au}+\mathrm{Au} \\
(\mathrm{Central} \mathrm{20 \% )}\end{array}$ & $p+p$ & $d+A u$ \\
\hline$>20 \mathrm{GeV}$ & $\begin{array}{l}10^{7} \text { jets } \\
10^{4} \text { photons }\end{array}$ & $\begin{array}{l}10^{6} \text { jets } \\
10^{3} \text { photons }\end{array}$ & $\begin{array}{l}10^{7} \text { jets } \\
10^{4} \text { photons }\end{array}$ \\
\hline$>30 \mathrm{GeV}$ & $\begin{array}{l}10^{6} \text { jets } \\
10^{3} \text { photons }\end{array}$ & $\begin{array}{l}10^{5} \text { jets } \\
10^{2} \text { photons }\end{array}$ & $\begin{array}{l}10^{4} \text { jets } \\
10^{3} \text { photons }\end{array}$ \\
\hline $\begin{array}{l}>40 \mathrm{GeV} \\
>50 \mathrm{GeV}\end{array}$ & $\begin{array}{l}10^{5} \text { jets } \\
10^{4} \text { jets }\end{array}$ & $\begin{array}{l}10^{4} \text { jets } \\
10^{3} \text { jets }\end{array}$ & $\begin{array}{l}10^{4} \text { jets } \\
10^{4} \text { jets }\end{array}$ \\
\hline
\end{tabular}

The goal of sPHENIX is to collect a large sample of unbiased jet events in heavy ion collisions and polarized proton collisions to study the properties of the QGP. Based on the current performance of the RHIC accelerator and 20 weeks of physics running, estimates have been made on the number of jet events that are expected under different conditions. These rate are rates summarized in Table 1. Improvements in the performance of the accelerator are expected to result in larger jet samples then these estiamtes.

\section{The sPHENIX Detector}

The sPHENIX detector will replace the current PHENIX detector with a new detector consisting of a super-conducting solenoid magnet, hadronic and electromagnetic calorimetry and charged particle tracking. A conceptual design of the proposed detector is shown in Figure 2. A detailed description of the proposed sPHENIX detector can be found in [2].

The sPHENIX detector will provide full $2 \pi$ coverage in $\phi$ and $-1.1<\eta<1.1$ coverage in pseudorapidity. The design of the sPHENIX detector is driven by the physics measurements in the sPHENIX experiment which sets the requirements for the detector resolutions. In heavy ion collisions the underlying events produce a large number of particles that deposit energy in the calorimeter. Various algorithms have been developed to subtract this background energy from the total jet energy, but fluctuations in the background energy limit the jet energy resolution and sets the energy resolution for the calorimeters. This leads to an energy resolution requirement of $\sim 100 \% \sqrt{E}$ for the hadronic calorimeter and $\sim 15 \% \sqrt{E}$ for the electromagnetic calorimeters. These requirements are also consis- 


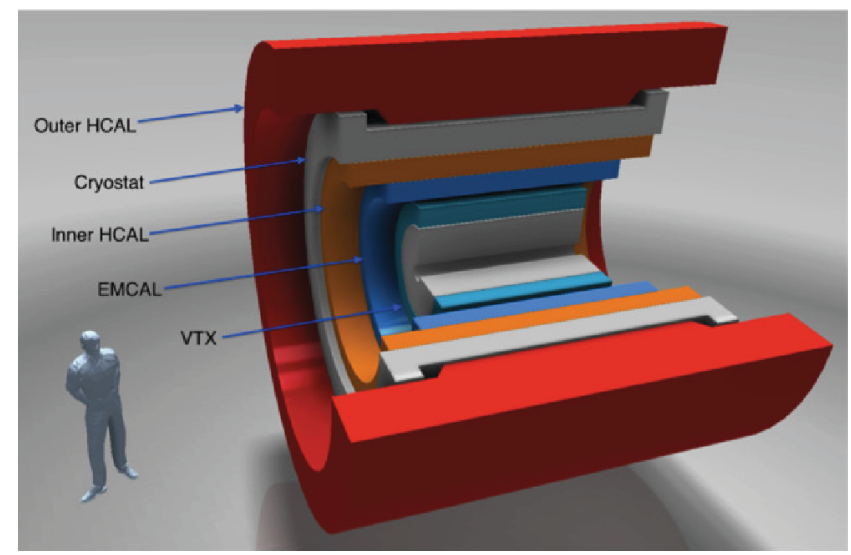

Figure 2: A conceptual drawing of the proposed sPHENIX detector showing the inner tracking, calorimetry and super-conducting solenoid. Not shown is the support structure and auxiliary infrastructure.

tent with other physics measurements such as direct photon and $\gamma$-jet correlations that can be measured in heavy ion collisions. The high multiplicity on heavy ion collisions require a segmentation of $\delta \eta \times \delta \phi \sim 0.1 \times 0.1$ for the hadronic calorimeter and $\delta \eta \times \delta \phi \sim 0.025 \times 0.025$ for the electromagnetic calorimeter.

In order to tag jet events with heavy quarks, a tracking system is required that can identify charged particles coming from the displaced decay vertices. For sPHENIX, the PHENIX VTX detector will be reconfigured and three additional layers of silicon tracking will be added to provide tracking over the full acceptance of the sPHENIX detector.

\subsection{The BaBar Solenoid}

The PHENIX collaboration has acquired the BaBar super-conducting solenoid for the sPHENIX detector. The solenoid generates a $1.5 \mathrm{~T}$ field that is homogeneous in the center, with the cryostat having an inner radius of $140 \mathrm{~cm}$ and outer radius of $170 \mathrm{~cm}$. With a total length of $385 \mathrm{~cm}$, it covers the full $|1.1|<\eta$ coverage required by the sPHENIX physics goals. Work has started to stabilize the magnet for transport from it's current location at Stanford Linear Accelerator (SLAC) in Stanford California to Brookhaven National Laboratory by the end of 2014. Once the magnet arrives at BNL, it will undergo preliminary testing and have the stack modified for the sPHENIX detector configuration.

\subsection{The Hadronic Calorimeter}

The hadronic calorimeter (HCal) design consists of two segments, inner and outer. A radial slice of the conceptual detector is shown in Figure 3. Both segments are comprised of tilted absorber plates and scintillating tiles with wave-shifting fibers embedded in the tile. The plates of the inner and outer segments run along the $\mathrm{Z}$ direction (beam line direction) and are tilted in opposite directions with a tilt angle $\left(\sim \pm 5^{\text {circ }}\right)$ such that a particle traveling from the interaction point will traverse 5 plates in the absence of any bending in the magnetic field. The total depth of the HCal is $\sim 5 \lambda_{a b s}$ with approximate $1 \lambda_{a b s}$ in the inner segment and $4 \lambda_{a b s}$ in the outer segment. Both the inner and outer segments are 


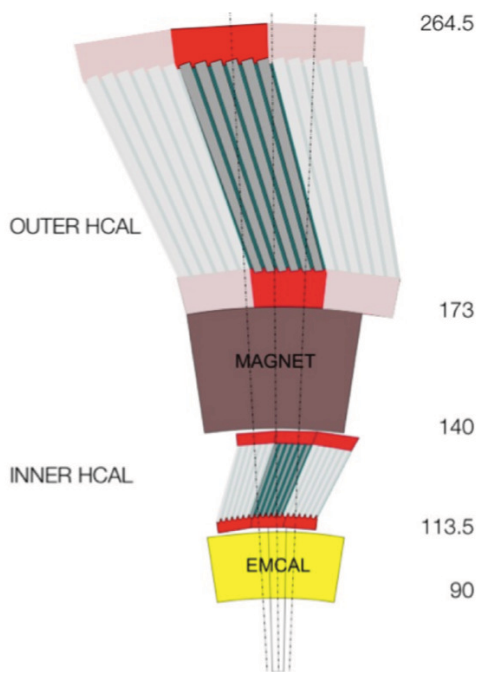

Figure 3: Conceptual design of the sPHENIX HCal showing the location of the inner and outer HCal sections. The outer HCal absorber is steel and acts as the flux return for the magnetic field.

divided into 22 towers in $\eta$ and 64 towers in $\phi$. The absorber for the inner segment will be stainless steel and the outer segment absorber will be steel, which will also serve as the magnetic flux return. Readout of the wave-shifting fibers will be with silicon photo-multipliers (SiPMs).

\subsection{The Electromagnetic Calorimeter}

For the electromagnetic calorimeter (EMCal) the SPACAL design that has been developed for the STAR upgrade and EIC detector[3] has been chosen. The detector is constructed from modular blocks of scintillating fibers embedded in a tungsten powder and epoxy. Prototype modules that have been developed for STAR and EIC are shown in Figure 4. Light mixing block on the end of the fibers are readout with multiple SiPMs, with the size of the mixing block defining the tower dimensions. For sPHENIX, there will be 96 towers in $\eta$ and 256 towers in $\phi$ each $\sim 2 \times 2 \mathrm{~cm}^{2}$, for a total of 24576 towers.

\subsection{Central Tracking}

The sPHENIX tracking system will consist of 5 layers of silicon tracking located inside the solenoid. The first 2 layers will be the current PHENIX VTX pixel layers reconfigured and expanded to provide complete $2 \pi$ in $\phi$. An additional 3 layers of silicon strip detectors will be built to track charged particles out to a radius of $60 \mathrm{~cm}$. The ladders in the additional layers will be built from single sided silicon sensors with $62.5 \mu \mathrm{m}$ spacing to achieve a momentum resolution of $\delta p / p \approx 2 \%$, and a DCA resolution of $<100 \mu \mathrm{m}$. Work is in progress to optimize the final tracking design.

\section{Projected Performance}

A GEANT-4 based simulation of the sPHENIX detector has been performed to evaluate the capabilities of the new sPHENIX detector and optimize both the design and physics capabilities of the 


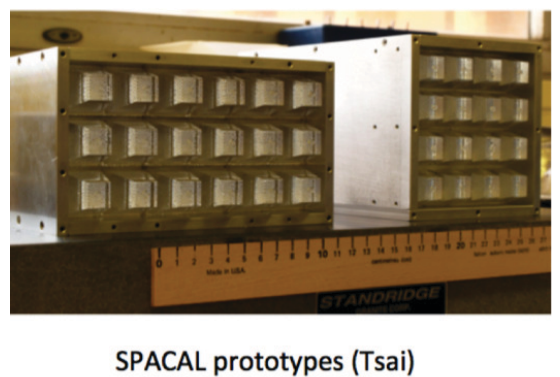

Figure 4: Prototype SPACAL modules used for performance measurements at the FNAL Test Beam Facility [3].

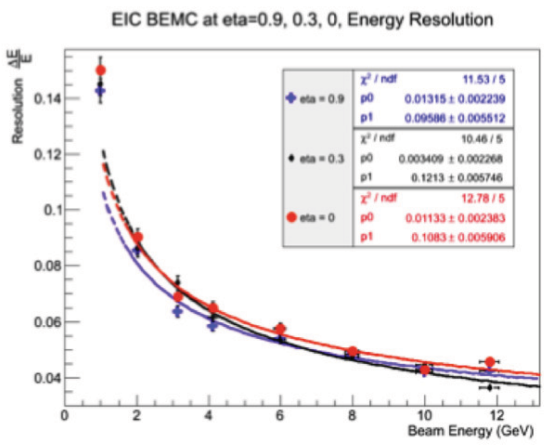

Figure 5: Energy resolution of EIC calorimeter for electrons vs energy for different angles [3].

detector. One challenge is the ability to separate real jets from fake jets in heavy ion collisions. Monte Carlo studies have been done to study the performance of jet reconstruction techniques [4] using HIJING events. Preliminary results show that for for a cone radius of 0.2 real jets dominate for jets with a $E_{T}>20 \mathrm{GeV}$ as shown in Figure 6 and increases to $30 \mathrm{GeV}$ for a cone radius of 0.4 as seen in Figure ??. \%includegraphics[width $=5 \mathrm{~cm}$,clip]jetstudies $-2 \%$ captionThe composition of the jet spectrum in central $\mathrm{Au}+\mathrm{Au}$ collisions based on 750M minimum bias HIJING events.

The primary motivation for studying heavy flavor jets in heavy ion collisions is to understand the mechanism for energy loss of the partons in the medium. Currently the PHENIX and STAR programs are making important measurements using semilpetonic decays of $D$ and $B$ mesons. The precision tracking and reconstruction of displaced vertices in sPHENIX will expand the understanding of energy loss in the medium by having the ability to reconstruct full jets with a heavy flavour tag. Figure 7 shows the expected rate of heavy quark events as a function of $p_{T}$ based on perturbative QCD calculations.

\section{Conclusions}

A proposal to upgrade the PHENIX detector called sPHENIX has been submitted to the U.S. Department of Energy which replaces the current PHENIX central arm spectrometer with a new detector based on the BaBar super-conducting solenoid, hadronic and electromagnetic calorimetry and 

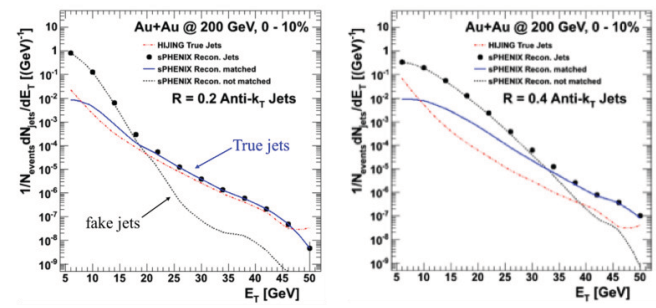

Figure 6: The composition of the jet spectrum in central $\mathrm{Au}+\mathrm{Au}$ collisions based on $750 \mathrm{M}$ minimum bias HIJING events. the full spectrum is shown as the solid points. The spectrum of those jets that are successfully matched is shown in blue curve. The jets which are not matched are known as fake jets and the spectrum of those jets is shown as the black dashed curve. The plot on the left is for a cone cut of $\mathrm{R}=0.2$ and real jets dominate fake jets above $20 \mathrm{GeV}$. The plot on the left is for a cone cut $\mathrm{R}=$ 0.4 and real jets dominate fake jets above $30 \mathrm{GeV}$.

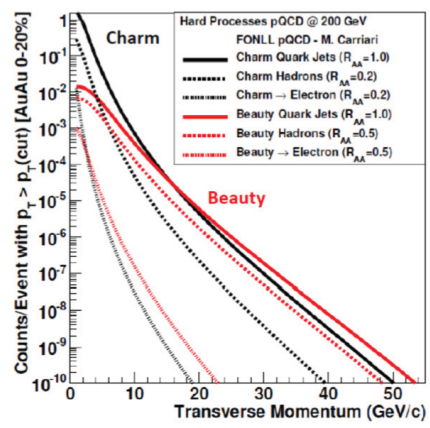

Figure 7: FONLL calculations [5] for heavy flavor jets, fragmentation hadrons and decay electrons as a function of transverse momentum. The rates have been scales to correspond to counts with $p_{T}>p_{T}(c u t)$ for Au-Au 0-20\% central collisions.

precision tracking. The sPHENIX detector will allow for a detailed study of the properties of the QGP through the measurement of jets in the heavy ion collisions at RHIC. The current schedule has sPHENIX construction starting in 2016 and being installed into the PHENIX experimental hall starting in 2017 and completed for first data taking in 2020.

\section{References}

[1] A. Majumder, B. Muller, X.N. Wang, Physical Review Letters (99), 192301 (2007)

[2] C. Aidala, et. al. (PHENIX Collaboration), arXiv:1207.6378v1, (2012)

[3] O.Tsai et. al., To Be Published in CALOR14 Proceedings, (2014)

[4] J.A. Hanks et. al., Physical Review C 86, 024908(2012)

[5] M. Cacciari, private communications. 